\title{
Non-equilibrium steady state of sparse systems
}

\author{
Daniel Hurowitz and Doron Cohen \\ Department of Physics, Ben-Gurion University of the Negev, P.O.B. 653, Beer-Sheva 84105, Israel
}

PACS

\begin{abstract}
A resistor-network picture of transitions is appropriate for the study of energy absorption by weakly chaotic or weakly interacting driven systems. Such "sparse" systems reach a novel non-equilibrium steady state (NESS) once coupled to a bath. In the stochastic case there is an analogy to the physics of percolating glassy systems, and an extension of the fluctuationdissipation phenomenology is proposed. In the mesoscopic case the quantum NESS might differ enormously from the stochastic NESS, with saturation temperature determined by the sparsity. A toy model where the sparsity of the system is modeled using a log-normal random ensemble is analyzed.
\end{abstract}

The study of systems with non-equilibrium steady state (NESS) has become active in recent years 1 , 6], involving various generalizations of linear response theory (LRT) and of the associated fluctuation-dissipation relation (FDR) 7 13. The paradigm for NESS (Fig,1a) is a system that is coupled to two equilibrated reservoirs, "A" and "B", which are characterized by different temperatures $T_{A}$ and $T_{B}$. Hence the NESS of the system is not canonical, and it cannot be characterized by a well-defined equilibrium temperature. A particular case of special interest is obtained if one reservoir (call it "A") is replaced by a stationary driving source, while the relaxation is provided by a bath (call it "B") that has some finite temperature $T_{B}$. This is still the same paradigm because formally the driving source "A" can be regarded as a bath that has an infinite temperature $T_{A}=\infty$.

Stochastic modeling.- The simplest modeling of NESS is obtained by considering a system that has energy levels $\left\{E_{n}\right\}$ with transition rates

$$
\mathcal{W}_{n m}=w_{n m}^{\varepsilon}+\frac{2 w_{n m}^{\beta}}{1+\mathrm{e}^{\left(E_{n}-E_{m}\right) / T_{B}}}
$$

where $w_{n m}^{\varepsilon}$ and $w_{n m}^{\beta}$ are the elements of symmetric matrices. The first term describes the transitions that are induced by the $T_{A}=\infty$ driving source. The second term describes the bath induced transitions, with ratio $\mathrm{e}^{\left(E_{n}-E_{m}\right) / T_{B}}$ of $n \Leftrightarrow m$ transitions, as required by detailed balance considerations. The dynamics of the population probabilities $\boldsymbol{p}=\left\{p_{n}\right\}$ is described by a rate equation

$$
\frac{d p_{n}}{d t}=\sum_{m}\left[\mathcal{W}_{n m} p_{m}-\mathcal{W}_{m n} p_{n}\right]
$$

This equation can be written schematically as $\dot{\boldsymbol{p}}=\mathcal{W} \boldsymbol{p}$, see remark a]. The steady state is determined from the matrix equation $\mathcal{W} \boldsymbol{p}=0$. In the presence of driving the detailed balance is disturbed leading in general to a noncanonical NESS.

Sparse systems .- In recent studies 14 16 our interest was focused on a class of driven systems for which the matrix $\left\{w_{n m}^{\varepsilon}\right\}$ is sparse. By this we mean that the transition rates are characterized by a log wide (say log normal) distribution. In other words, the majority of elements are small, while the large elements constitute a small fraction, $s \ll 1$. A system of current experimental interest is described in [16]: an optical billiards with vibrating walls, where the energy absorption rate (EAR) of the cold atoms is affected by the sparsity of the perturbation matrix, which is controlled either by the degree of chaoticity or by the strength of the inter-atomic interactions. Yet a simpler example concerns the absorption of low frequency irradiation by small metallic grains [15], where the transitions are predominately between neighboring energy levels, and the sparsity is determined by the level spacing statistics.

Beyond LRT .- In the absence of a bath the rate equation $\dot{\boldsymbol{p}}=\mathcal{W} \boldsymbol{p}$ generates diffusion in energy space. In this context it is useful to picture the levels $n$ as "sites" in space, or as the "nodes" of a network, and the $w_{n m}^{\varepsilon}$ as "connectors" (Fig.1p). The calculation of the diffusion coefficient $D$ is exactly as the calculation of electrical conductivity. For example, when connecting $N$ nodes in series (as in the Chain model to be discussed later), the "conductivity" is $D=\left[(1 / N) \sum_{n}\left(1 / w_{n}\right)\right]^{-1}$. The adap- 
tation of the resistor network picture to the calculation of $D$ is termed semi-linear response theory (SLRT) because $D$ is a semi-linear function of the couplings $w_{n m}$. This means that $D[c \boldsymbol{w}]=c D[\boldsymbol{w}]$, but there is no additivity, $D\left[\boldsymbol{w}+\boldsymbol{w}^{\prime}\right] \neq D[\boldsymbol{w}]+D\left[\boldsymbol{w}^{\prime}\right]$. Due to the sparsity, the result is very similar to that of percolating or disordered resistor-networks 17.

Outline.- Considering the coupling of a "sparse" system to a bath, our expectation is to have, as the driving becomes stronger, a crossover from an LRT canonical-like NESS to a novel non-canonical NESS, with the possibility of remarkable quantum-mechanical fingerprints. Specifically our objective is to calculate the energy absorption rate (EAR) of a driven "sparse" system, taking into account the presence of a surrounding environment. Below we (i) introduce the FDR phenomenology of calculating the EAR, which requires a notion of effective NESS temperature; (ii) demonstrate this phenomenology for the simplest toy model, obtaining explicit expressions for both $T$ and $D$; and (iii) discuss the quantum case, highlighting the existence of a saturation temperature $T_{\infty}$ that is determined by the sparsity.

FDR phenomenology.- In the standard textbook presentation it is assumed that the system reaches a canonical-like state with a well-defined temperature $T_{\text {sys }}$. The driving induces diffusion with coefficient $D$ in energy space 18 22. From $\dot{E}=\sum_{n} E_{n} \dot{p}_{n}$, substituting $\dot{\boldsymbol{p}}=\mathcal{W} \boldsymbol{p}$, it follows (see appendix) that the EAR is

$$
\dot{\mathrm{W}}=\sum_{n, m}\left(E_{n}-E_{m}\right) w_{n m}^{\varepsilon} p_{m}=\frac{D}{T_{\mathrm{sys}}}
$$

with

$$
D_{[\mathrm{LRT}]}=\frac{1}{2} \overline{\sum_{n} w_{n m}^{\varepsilon}\left(E_{n}-E_{m}\right)^{2}}
$$

where the overline indicates canonical averaging over the initial state. In complete analogy it is straightforward to show (see appendix), that the rate of cooling due to the interaction with the bath can be written as

$$
\dot{\mathrm{Q}}=-\sum_{n, m}\left(E_{n}-E_{m}\right) w_{n m}^{\beta} p_{m}=\frac{D_{B}}{T_{B}}-\frac{D_{B}}{T_{\mathrm{sys}}}
$$

where the first term is due to the imbalance of upward and downward transition rates, while the second term is due to the non-uniformity of the probability distribution in energy space. The net rate of energy increase is $\dot{E}=\dot{W}-\dot{Q}$. At steady state $\dot{W}=\dot{Q}$, so a phenomenological determination of the steady state temperature $T_{\mathrm{sys}}$ is possible.

The essence of the above FDR phenomenology is the same as in Einstein's relation: the dissipation is related to the diffusion (in LRT the latter is determined by the fluctuations, e.g. the velocity-velocity correlation in Einstein's relation, hence the terminology). Our purpose below is to generalize the FDR. We emphasize in advance two issues: (a) The NESS might be non-canonical, so we have to define its effective temperature; (b) The diffusion coefficient is not necessarily determined by LRT.

The Chain model.- It is best to clarify the determination of $T_{\text {sys }}$ and $D$ by considering the simplest example of a "chain" with nearest-neighbor transitions only. With simplified indexing, Eq. (1) for $(n-1) \Leftrightarrow n$ transitions is written as $w_{n}+2 w^{\beta} /\left(1+\mathrm{e}^{ \pm \Delta_{0} / T_{B}}\right)$, where $\Delta_{0}$ is the level spacing, and $n=1,2, \cdots$. In contrast to $w^{\beta}$, which is the same for all transitions, the rates $w_{n}$ are characterized by a logarithmically wide distribution.

In the numerical example we have $N=25$ levels, with equal level spacing $\Delta_{0}=1$. The bath temperature is $T_{B}=10$. The bath induced transition rates are taken as $w^{\beta}=0.1$. The driving induced transition rates are log normally distributed. Namely, $w_{n}=\exp \left(x_{n}\right)$, where the $x_{n}$ have a Gaussian distribution with average $\mu$ and dispersion $\sigma$ that are determined such that the driving intensity is $\overline{w_{n}}=\varepsilon^{2}$, and the sparsity 16 is $s=\exp \left(-\sigma^{2}\right)$. The value $s \sim 1$ means that all the elements are comparable and well represented by their average. Sparsity means $s \ll 1$, for which the median differs by orders of magnitude from the algebraic average.

From the NESS equation $\mathcal{W} \boldsymbol{p}=0$ we determine the occupation probabilities $p_{n}$ as in Fig.2, and then calculate the EAR via either Eq. (3) or Eq. (5) as in Fig 3 . In the absence of driving, the steady state is canonical with a welldefined temperature $T_{B}$. In the presence of driving, the state is generally not canonical. Consequently, we can formally associate a different microscopic temperature $T_{n m}$ for each pair of coupled levels via the defining equation

$$
\frac{p_{n}}{p_{m}}=\exp \left(-\frac{E_{n}-E_{m}}{T_{n m}}\right)
$$

For the Chain model we use the simpler indexing $T_{n}$, and the NESS equation $\mathcal{W} \boldsymbol{p}=0$ takes the form $p_{n} / p_{n-1}=$ $\mathcal{W}_{n, n-1} / \mathcal{W}_{n-1, n}$. Assuming $\Delta_{0} \ll T_{B}$ we deduce that the microscopic temperature of the $n t h$ transition is given by the expression

$$
T_{n}=\left[\frac{w_{n}+w^{\beta}}{w^{\beta}}\right] T_{B}
$$

Effective NESS temperature.- We define the NESS temperature $T_{\mathrm{sys}}$ such that the phenomenological FDR Eq. (5) still holds. For the Chain model the bath induced diffusion is $D_{B}=w^{\beta} \Delta_{0}^{2}$, and it is straightforward to show (see appendix) that $T_{\mathrm{sys}}$ should be defined as the harmonic average over $T_{n}$, i.e.

$$
T_{\mathrm{sys}}=\left[\overline{\left(\frac{1}{T_{n}}\right)}\right]^{-1}=\left[\overline{\left(\frac{w^{\beta}}{w^{\beta}+w_{n}}\right)}\right]^{-1} T_{B}
$$

For numerical results see (Fig.4.5). As the driving becomes stronger, the temperature becomes higher, with the asymptotic behavior $T_{\mathrm{sys}} \propto \varepsilon^{2}$ as implied by Eq. 8. 
LRT-SLRT crossover.- We continue with the stochastic Chain model and find from Eq. (3), substituting Eq. (7) in Eq. 25 of the appendix, that the EAR is given by the expression

$$
\dot{\mathbf{W}}=\left[\overline{\left(\frac{w_{n}}{w^{\beta}+w_{n}}\right)}\right] \frac{D_{B}}{T_{B}} \equiv \frac{D}{T_{\mathrm{sys}}}
$$

We define the driving induced diffusion $D$ such that the phenomenological FDR still holds:

$$
D=\left[\overline{\left(\frac{w_{n}}{w^{\beta}+w_{n}}\right)}\right]\left[\overline{\left(\frac{1}{w^{\beta}+w_{n}}\right)}\right]^{-1} \Delta_{0}^{2}
$$

In the limit of very weak and very strong driving we respectively get

$$
\begin{aligned}
D_{\text {[LRT }]} & =\overline{w_{n}} \Delta_{0}^{2} \\
D_{[\mathrm{SLRT}]} & =\left[\overline{1 / w_{n}}\right]^{-1} \Delta_{0}^{2}
\end{aligned}
$$

The LRT result could be obtained from the traditional Kubo formalism, while the SLRT prediction reflects a network that consists of connectors that are connected in series. Note that if all the $w_{n}$ are comparable, then $D \approx D_{[\mathrm{LRT}]} \approx D_{\text {[SLRT] }}$. But if the $w_{n}$ have a log-wide distribution, the agreement with LRT is achieved only if the $w_{n}$ are all much smaller than $w^{\beta}$. For strong driving, both $D$ and $T_{\text {sys }}$ are $\propto\left[\overline{1 / w_{n}}\right]^{-1}$, and hence $\propto \varepsilon^{2}$, as expected from the SLRT resistor-network phenomenology. In this limit their ratio approaches the bath limited value

$$
\dot{\mathrm{W}}_{\infty}=\frac{D_{B}}{T_{B}}
$$

which is implied by Eq. 5 .

Quantum modeling.- It should be clear that SLRT applies whenever the transport is modeled using a resistor network. Thus it might have applications, e.g., in statistical mechanics and biophysics. But the original motivation for SLRT came from mesoscopics, where the quantum nature of reality cannot be ignored. In this context the Hamiltonian contains a driving term $f(t) V$, and the transition rates $w_{n m}^{\varepsilon} \propto\left|V_{n m}\right|^{2}$ between levels are determined by the Fermi-Golden-Rule (FGR). For weakly chaotic or weakly interacting systems $V_{n m}$ is typically sparse and textured. Assuming that $f(t)$ has zero average, and correlation function $\left\langle f(t) f\left(t^{\prime}\right)\right\rangle=\varepsilon^{2} \delta\left(t-t^{\prime}\right)$, the rate equation Eq. (2) is replaced by the quantum Master equation

$$
\frac{d \rho}{d t}=-i\left[\mathcal{H}_{0}, \rho\right]-\frac{\varepsilon^{2}}{2}[V,[V, \rho]]+\mathcal{W}^{\beta} \rho
$$

where the second and third terms correspond to the driving source and to the bath as in Eq.(1). This equation is of the form $\dot{\rho}=\mathcal{W} \rho$. It is identical with $\dot{\boldsymbol{p}}=\mathcal{W} \boldsymbol{p}$ of Eq. 22 if the off-diagonal terms are ignored, where

$$
w_{n m}^{\varepsilon}=\varepsilon^{2}\left|V_{n m}\right|^{2}
$$

Note that $\mathcal{W}^{\beta}$ induces dephasing of the off-diagonal elements, which we assume to be minimal in the "quantum" case: see technical details below.

Some technical details with regard to Eq. 114 are in order (can be skipped in first reading). In the energy basis, $\mathcal{H}_{0}$ is a diagonal matrix with energy levels $E_{n}$. The state of the system is represented by the probability matrix, which can be rewritten as a column vector $\rho \mapsto\left(p_{n} ; \rho_{\nu \mu}\right)$, composed of the diagonal probabilities and the off-diagonal coherences. Consequently, the master equation takes the form $\dot{\rho}=\mathcal{W} \rho$, with the super operator

$$
\mathcal{W}=\left(\begin{array}{cc}
\mathcal{W} & \Lambda^{\dagger} \\
\Lambda & \mathcal{W}^{\perp}
\end{array}\right)
$$

The matrix $\mathcal{W}$ is given by Eq. (1) with $w_{n m}^{\varepsilon}=\varepsilon^{2}\left|V_{n m}\right|^{2}$. The definition of $\Lambda$ is implied by the second term in Eq. 14. In particular we note that

$$
\begin{aligned}
\mathcal{W}_{\nu \mu, \nu \mu}^{\perp} & =i \Delta_{\nu \mu}-\gamma_{\nu \mu}-\gamma_{\beta} \\
\Lambda_{n, \nu \mu} & =\varepsilon^{2} V_{n \nu} V_{\mu n}, \quad \text { for } \nu, \mu \neq n
\end{aligned}
$$

We use the common notations $\Delta_{\nu \mu}=E_{\nu}-E_{\mu}$, and $\gamma_{\nu \mu}=$ $\left(\varepsilon^{2} / 2\right)\left[\left(V^{2}\right)_{\nu \nu}+\left(V^{2}\right)_{\mu \mu}\right]$. For simplicity, we assume that the bath induced dephasing $\gamma_{\beta}=w^{\beta}+\gamma_{\varphi}$ is the same for all the coherences.

In the absence of driving Eq. (14) is the well known Pauli master equation leading to canonical equilibrium. In the numerics we assume in the "quantum" case minimal dephasing, which means $\gamma_{\varphi}=0$. More generally we may have an extra "pure dephasing" effect which is represented by $\gamma_{\varphi}>0$ as in the familiar NMR context.

The stochastic (FGR) picture applies if the coherences become negligible. By inspection, it might happen for two reasons. One possibility is that there is strong pure dephasing effect $\left(\gamma_{\varphi} \gg w^{\varepsilon}\right)$ that suppresses the coherences. Then one simply recovers the stochastic rate equation for the occupation probabilities. The second possibility is to have $w^{\varepsilon}$ much smaller compared with the level spacing $\Delta_{0}$. The latter is the traditional assumption in atomic physics, and can be regarded as "microscopic circumstances". But in "mesoscopic circumstances" $\Delta_{0}$ might be small, and the validity of FGR is not guaranteed.

Quantum NESS.- The NESS is obtained by solving the equation $\mathcal{W} \rho=0$. Looking at Fig. 4.5 we see that in the "quantum" case there is a saturation temperature $T_{B}<T_{\infty}<\infty$ that depends on the sparsity $s$. Consequently there is a premature saturation of the EAR at

$$
\dot{\mathrm{W}}_{\infty}=\frac{D_{B}}{T_{B}}-\frac{D_{B}}{T_{\infty}}
$$

as seen in Fig 3 . Note [b. Only in the non-sparse limit do we recover quantum-to-classical correspondence.

One would like to understand how $T_{\infty}$ emerges. For this purpose we regard Eq. (14) for $\rho_{n n^{\prime}}$ as a Fokker-Planck equation, where $n$ is the momentum. In the absence of sparsity, $V_{n n^{\prime}}$ can be interpreted as the matrix representation of the position coordinate, and its eigenstates are 
extended in $n$. But if $s \ll 1$, then $V_{n n^{\prime}}$ is like off-diagonal disorder, and its eigenstates $r$ become localized in $n$, with energies $\langle E\rangle_{r}$ that are no longer identical. If the driving is very strong, the master equation implies that the NESS becomes a mixture of the eigenstates $r$ with weights

$$
p_{r} \propto \exp \left[-\frac{\langle E\rangle_{r}}{T_{\text {mix }}}\right]
$$

In the sparse limit the $n$ and the $r$ bases essentially coincide, and accordingly $T_{\infty} \sim T_{\text {mix }} \sim T_{B}$. This is confirmed numerically in Fig 6 .

In order to further illuminate the variation of the saturation temperature as a function of $s=\exp \left(-\sigma^{2}\right)$, we have added in Fig 6 a lower bound estimate for $T_{\infty}$ which is deduced as follows $[\mathrm{c}$ : Considering an energy range $\Delta\left(E_{n}\right)$ of interest, one observes that as $\sigma$ is decreased, the $\langle E\rangle_{r}$ occupy a smaller range $\Delta\left(E_{r}\right)$, and eventually in the non-sparse limit they are quasi-degenerate. The $p_{r}$ distribution is characterized by some $T_{m i x} \geq T_{B}$. Consequently the stretched distribution $p_{n}$ is characterized by a higher temperature $T_{\infty} \geq\left[\Delta\left(E_{n}\right) / \Delta\left(E_{r}\right)\right] T_{B}$. Hence the saturation temperature increases for smaller $\sigma$, and diverges in the non-sparse limit.

Experimental Signature.- The thermodynamic definition of temperature which we have adopted via Eq.(5) is related to the heat flow between two bodies: it is the same concept which is reflected in the phrasing of "the zeroth law of thermodynamics" and in the associated definition of empirical temperature. It should be contrasted with an optional statistical mechanics perspective that relates the temperature to the fluctuations, as in the paradigmatic Brownian motion studies of Einstein. Once $T_{\text {sys }}$ of Eq. (5) is determined through a heat flow measurement, it is possible to determine experimentally also the $D$ in Eq. (3) through an EAR measurement. Then it is possible to explore the LRT to SLRT crossover of this absorption coefficient Eq. 11]-Eq. 12. This crossover is reflected in having a wide range (many decades) over which the EAR varies as in Fig. 3. It is important to realize that the unlimited increase of temperature in the classical case does not have by itself a reflection in the EAR, because classically the EAR always saturates to a bath limited value as determined by Eq.13. In contrast to that, quantum mechanically there is a premature saturation, as expected from Eq. 19), reflecting the sparsity of the system. This effect might be useful for the detection of a classical to quantum transition with non-equilibrium measurements.

Discussion.- The "sparse" NESS resembles that of a glassy system 11]: In both cases, one has to distinguish between "microscopic" and "macroscopic" time scales, rates, and temperatures. Eq. (3) and Eq. (5) establish a diffusion-dissipation relation involving a "macroscopic" temperature $T$ that might be much lower compared with the microscopic temperatures $T_{n}$. The diffusion is driven by the fluctuations of the sources, but it is not the LRT (Kubo) formula which should be used in order to determine $D$, but rather a resistor network SLRT calculation.
A closely related chain of works regarding NESS, concerns mixed phase-space of periodically driven systems 4 6, where the problem is reduced to the study of a stochastic rate equation. Our work differs in three respects: (1) The sparsity may arise even for quantized chaotic nonmixed systems, implying a glassy type of NESS; (2) We assume a stationary driving source instead of a strictly periodic driving; (3) The master equation approach has allowed us to consider novel mesoscopic circumstances in which the quantum NESS differs enormously from the stochastic prediction.

The influence of quantum coherence on the NESS is remarkable. Due to the localization of the eigenstates in energy space, we found that for strong driving the temperature saturates to a finite value that reflects the sparsity of system. This should be contrasted with the traditional prediction of unbounded temperature.

Appendix.- For the convenience of the reader we concentrate here the technical details that concern the derivation of the FDR phenomenology. The energy of the system is $E=\sum_{n} p_{n} E_{n}$, and its rate of change is $\dot{E}=\sum_{n} E_{n} \dot{p}_{n}$. The equation for $\dot{p}_{n}$ includes a driving source term and a bath term. Accordingly we write $\dot{E}=\dot{W}-\dot{Q}$, where the two terms are interpreted as the rate of heating due to the driving (equals the EAR), and the rate of cooling due to the bath (in steady state equals the EAR too). From the master equation it follows that the expression for $\dot{Q}$, both in the stochastic and in the quantum case, is given by the first equality of Eq. (5). This expression can be written as the sum of a term that originates from the asymmetry of $w_{n m}^{\beta}$, and a term that originates from the non-uniformity of the $p_{n}$. Defining $\bar{p}_{n m}=\left(p_{n}+p_{m}\right) / 2$ we get

$$
\begin{aligned}
w_{n m}^{\beta}-w_{m n}^{\beta} & =\left[2 \tanh \left(-\frac{E_{n}-E_{m}}{2 T_{B}}\right)\right] \bar{w}_{n m}^{\beta} \\
p_{n}-p_{m} & =\left[2 \tanh \left(-\frac{E_{n}-E_{m}}{2 T_{n m}}\right)\right] \bar{p}_{n m}
\end{aligned}
$$

At high temperatures one can approximate the $\tanh ()$ by linear functions leading to

$$
\begin{aligned}
\dot{\mathrm{Q}} & =\frac{1}{2} \sum_{n, m} \bar{p}_{n m} \frac{\bar{w}_{n m}^{\beta}}{T_{B}}\left(E_{n}-E_{m}\right)^{2} \\
& -\frac{1}{2} \sum_{n, m} \bar{p}_{n m} \frac{\bar{w}_{n m}^{\beta}}{T_{n m}}\left(E_{n}-E_{m}\right)^{2}
\end{aligned}
$$

The first expression is identified as $D_{B} / T_{B}$, where $D_{B}$ is the bath induced diffusion coefficient. The second expression is used to define the effective system temperature $T_{\mathrm{sys}}$, such that it takes the form $-D_{B} / T_{\mathrm{sys}}$.

In the stochastic case the effect of the driving can be treated using the same procedure. The expression for $\dot{W}$ is given by the first equality of Eq. (3), that is analogous to Eq. (5). Taking into account that $w_{n m}^{\varepsilon}$ unlike $w_{n m}^{\beta}$ is a symmetric matrix one obtains, in analogy to Eq. 24,

$$
\dot{\mathrm{W}}=\frac{1}{2} \sum_{n, m} \bar{p}_{n m} \frac{w_{n m}^{\varepsilon}}{T_{n m}}\left(E_{n}-E_{m}\right)^{2}
$$


If the state is strictly canonical with all the $T_{n m}$ equal the same number $T_{\text {sys }}$, then $\dot{\mathrm{W}}=D / T_{\text {sys }}$ where $D$ is given by Eq. (4). This is the standard LRT expression for the diffusion coefficient, leading to the linear result Eq. 111 in the case of near-neighbor transitions.

More generally $T_{\mathrm{sys}}$ is the effective temperature, and the equation $\dot{\mathrm{W}}=D / T_{\mathrm{sys}}$ is used to define the effective diffusion coefficient $D$, leading in the stochastic case to Eq. 10. This agrees with the linear result Eq. 11) for weak driving, and with the semi-linear result Eq. 12 for strong driving.

Acknowledgments: This research has been supported by the US-Israel Binational Science Foundation (BSF).

\section{REFERENCES}

[a] There is an implicit conservation of probability requirement $\sum p_{n}=1$. The diagonal elements of $\mathcal{W}$ are $-\Gamma_{n}$, with the decay rates $\Gamma_{n}=\sum_{m} \mathcal{W}_{m n}$.

[b] Note that Eq. (5) unlike Eq. (9) can be trusted also in the quantum analysis, because the last term in Eq. 14 does not couple diagonal and off-diagonal terms.

[c] The numerical demonstration in Fig.6 is done for pedagogical purpose, to clarify the reasoning with regard to the dependence of $T_{\infty}$ on $\sigma$. Obviously the numerics is dominated by the finite size truncation of energy space.

[1] J.R. Dorfman, An Introduction to Chaos in Nonequilibrium Statistical Mechanics. Cambridge University Press, Cambridge (1999).

[2] J.-P. Eckmann, C.-A. Pillet, L. Rey-Bellet, Commun. Math. Phys. 201, 657 (1999).

[3] H. Qian J. Phys. Chem. B 110, 15063-15074 (2006)

[4] H.-P. Breuer, W. Huber, F. Petruccione, Phys. Rev. E 61, 4883-4889 (2000).

[5] W. Kohn, J. Stat. Phys. 103, 417-423 (2001).

[6] D.W. Hone, R. Ketzmerick, W. Kohn, Phys. Rev. E 79, 051129 (2009). R. Ketzmerick, W. Wustmann, arXiv: 1005.0757

[7] G. Gallavotti, E.G.D. Cohen, Phys. Rev. Lett. 74, 2694 (1995). G. Gallavotti, E.G.D. Cohen, J. Stat. Phys. 80, 931-970 (1995).

[8] G. Crooks, Phys. Rev. E 60, 2721-2726 (1999).

[9] C. Jarzynski, Phys. Rev. Lett. 78, 2690 (1997).

[10] D.J. Evans, E.G.D. Cohen, G.P. Morriss, Phys. Rev. Lett. 71, 2401 (1993). D.J. Evans and D.J. Searles, Phys. Rev. E 50, 1645 (1994).

[11] J. Kurchan, Nature 433, 222 (2005).

[12] A. Crisanti, F. Ritort, J. Phys. A 36, R181-R290 (2003).

[13] T. Harada, S.-i. Sasa, Phys. Rev. Lett. 86, 3463-3466 (2001). Phys. Rev. E 73, 026131 (2006).

[14] D. Cohen, T. Kottos, H. Schanz, J. Phys. A 39, 11755 (2006). A. Stotland, R. Budoyo, T. Peer, T. Kottos, D. Cohen, J. Phys. A 41, 262001(FTC) (2008). A. Stotland, T. Kottos, D. Cohen, Phys. Rev. B 81, 115464 (2010).

[15] M. Wilkinson, B. Mehlig, D. Cohen, Europhys. Lett. 75, 709 (2006).

[16] A. Stotland, D. Cohen, N. Davidson, Europhys. Lett. 86, 10004 (2009). A. Stotland, L.M. Pecora and D. Cohen, Europhys. Lett. 92, 20009 (2010).
[17] A. Miller and E. Abrahams, Phys. Rev. 120, 745 (1960). V. Ambegaokar, B. Halperin, J.S. Langer, Phys. Rev. B 4, 2612 (1971).

[18] E. Ott, Phys. Rev. Lett. 42, 1628-1631 (1979). R. Brown, E. Ott, C. Grebogi, Phys. Rev. Lett. 59, 1173-1176 (1987).

[19] C. Jarzynski, Phys. Rev. E 48, 4340-4350 (1993).

[20] M. Wilkinson, J. Phys. A 21, 4021-4037 (1988).

[21] J.M. Robbins, M.V. Berry, J. Phys. A 25 L961-L965 (1992).

[22] D. Cohen, Annals of Physics 283, 175-231 (2000).

(a)

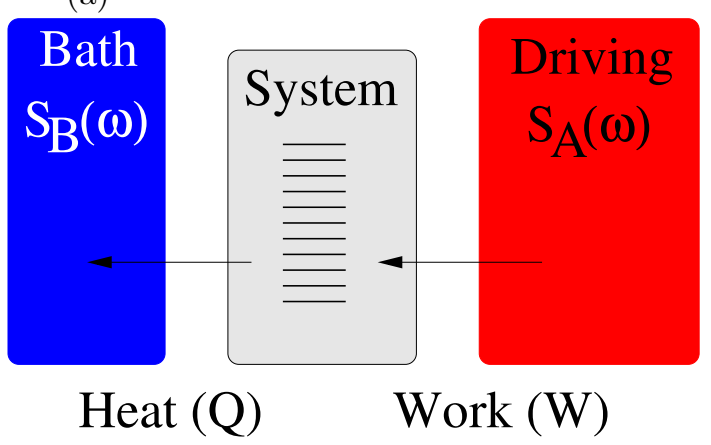

(b)

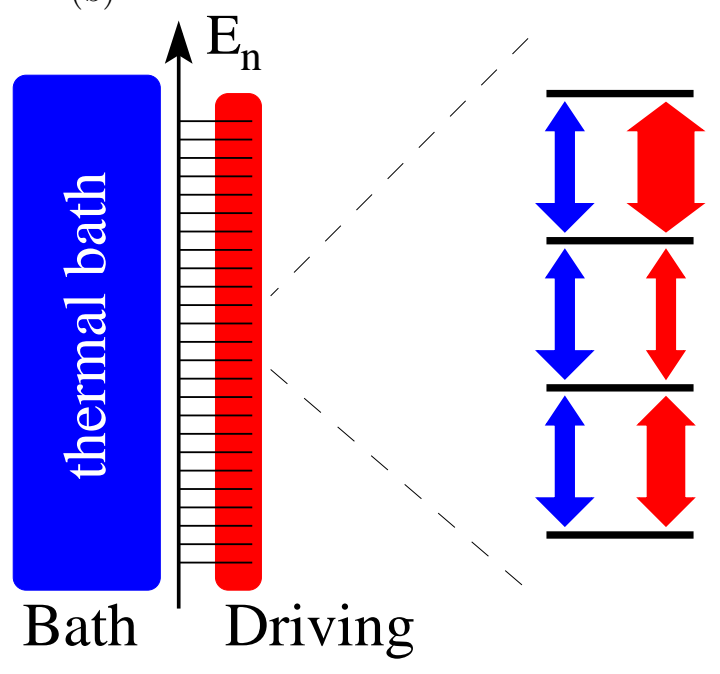

Fig. 1: (a) Block diagram of the model system. Both the driving source (A) and the bath (B) induce fluctuations that drive transitions. These fluctuations are characterized by a power spectrum $\tilde{S}(\omega)$. The driving source can be formally regarded as a bath that has an infinite temperature $T_{A}=\infty$, such that $\tilde{S}_{A}(-\omega)=\tilde{S}_{A}(\omega)$. In contrast to that $T_{B}$ is finite, and accordingly $\tilde{S}_{B}(-\omega) / \tilde{S}_{B}(\omega)=\exp \left(\omega / T_{B}\right)$. (b) Illustration of the Chain model. In the absence of a bath the driving induces transitions (red arrows) between levels $E_{n}$ of a closed system, leading to diffusion in energy space and hence heating. The diffusion coefficient $D$ can be calculated using a resistor network analogy. A NESS is reached due to the presence of a heat bath (blue arrows) that favors downward transitions. 


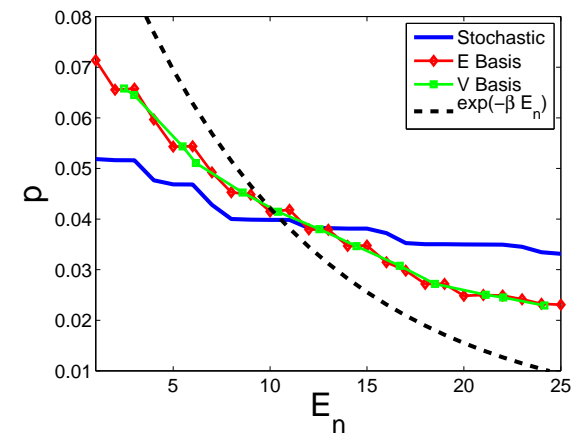

Fig. 2: The NESS occupation probabilities $p_{n}$ are plotted vs $E_{n}$ in the stochastic (blue) and quantum (red) cases. In the latter (quantum) case we plot also the occupation probabilities $p_{r}$ of the $V$ eigenstates versus $\langle E\rangle_{r}$. The sparsity is $s=10^{-5}$, and $\varepsilon=9.3$. We observe that the effective temperature predicted by the quantum master equation is lower compared with the stochastic approximation.

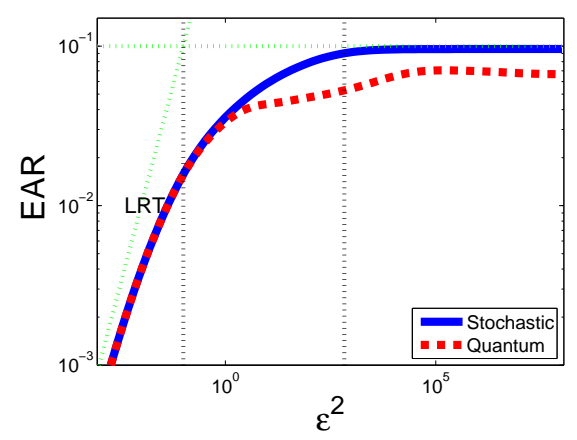

Fig. 3: The EAR $\dot{W}$ for the stochastic (solid blue) and for the quantum (dashed red) NESS. The sparsity is $s=10^{-5}$. The vertical lines are plotted at values of $\varepsilon$ for which the stochastic picture predicts a crossover: i.e. the $\varepsilon$ values for which $\overline{w_{n}}$ and $\overline{\left[1 / w_{n}\right]^{-1}}$ equal $w^{\beta}$.

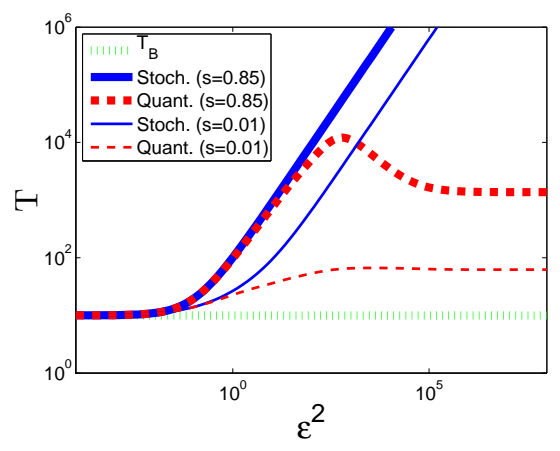

Fig. 4: The effective NESS temperature $T_{\mathrm{sys}}$ versus the driving intensity. Solid (blue) lines are for the stochastic NESS, while dashed (red) lines are for the quantum NESS. The dotted (green) line represents the temperature $T_{B}$ of the bath.
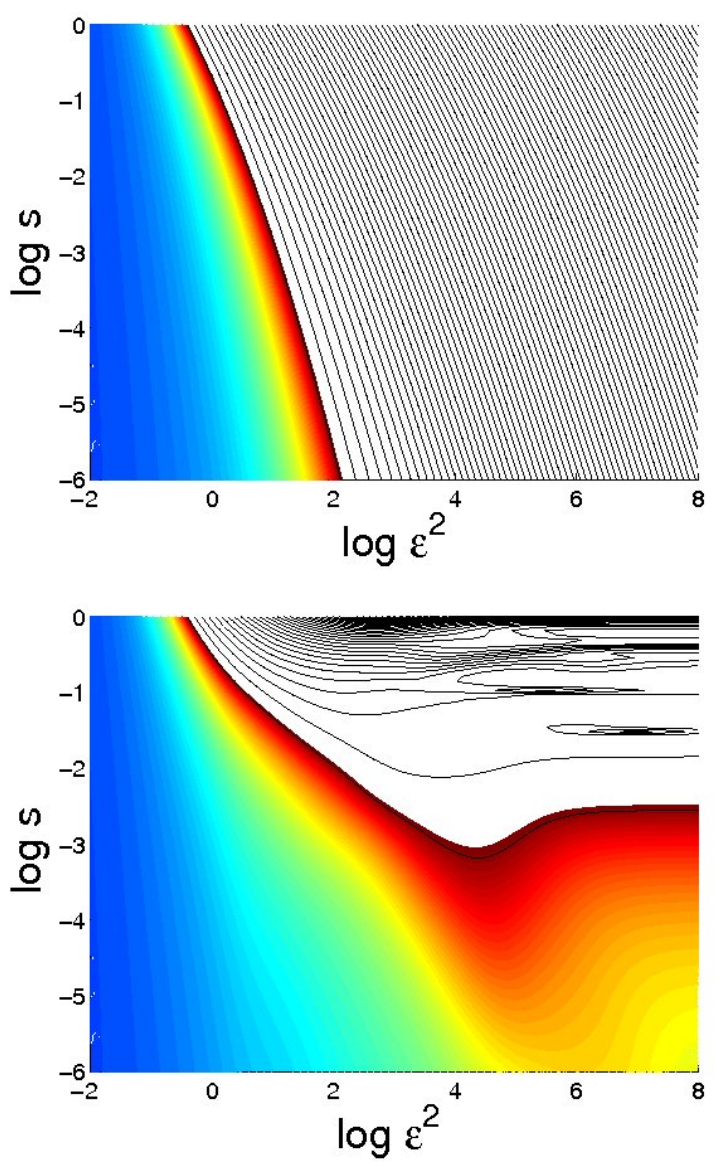

Fig. 5: The effective NESS temperature $T_{\text {sys }}$ in the stochastic case (upper panel) and in the quantum case (lower panel) is imaged for additional values of the sparsity. (Color online) Blue represents the bath temperature $T=10$, while red corresponds to $T=50$. For quantitative dependence see Fig 4

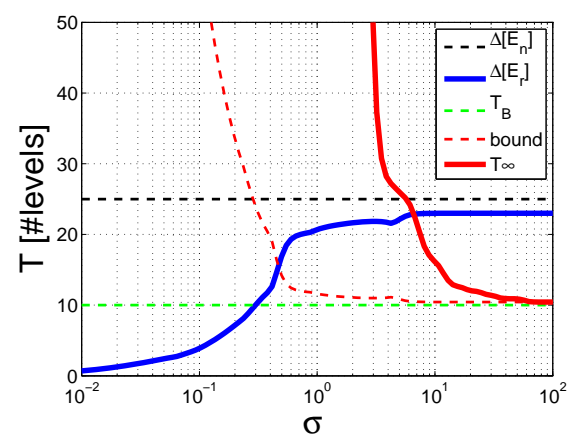

Fig. 6: The dependence of $T_{\infty}$ on the width $\sigma$ of the log-normal distribution. Note that the sparsity is $s=\exp \left(-\sigma^{2}\right)$. We confirm that $T_{\infty}$ is bounded from below by $\left[\Delta\left(E_{n}\right) / \Delta\left(E_{r}\right)\right] T_{B}$ (dashed red line), and tends to $T_{B}$ in the sparse limit. Here $\Delta\left(E_{n}\right)=25$ is the width of energy window in this numerical test, while $\Delta\left(E_{r}\right)$ is the length of the interval that contains the energies $\langle E\rangle_{r}$. 